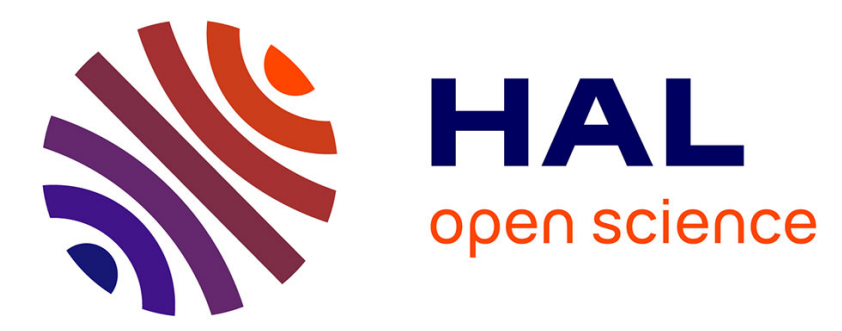

\title{
Automated markerless registration of point clouds from TLS and structured light scanner for heritage documentation
}

\author{
Jie Shao, Wuming Zhang, Nicolas Mellado, Pierre Grussenmeyer, Renju Li, \\ Yiming Chen, Peng Wan, Xintong Zhang, Shangshu Cai
}

\section{To cite this version:}

Jie Shao, Wuming Zhang, Nicolas Mellado, Pierre Grussenmeyer, Renju Li, et al.. Automated markerless registration of point clouds from TLS and structured light scanner for heritage documentation. Journal of Cultural Heritage, 2018, 32, pp.16-24. 10.1016/j.culher.2018.07.013 hal-01884798

\section{HAL Id: hal-01884798 https://hal.science/hal-01884798}

Submitted on 19 Nov 2019

HAL is a multi-disciplinary open access archive for the deposit and dissemination of scientific research documents, whether they are published or not. The documents may come from teaching and research institutions in France or abroad, or from public or private research centers.
L'archive ouverte pluridisciplinaire $\mathbf{H A L}$, est destinée au dépôt et à la diffusion de documents scientifiques de niveau recherche, publiés ou non, émanant des établissements d'enseignement et de recherche français ou étrangers, des laboratoires publics ou privés. 


\title{
1 Automated Markerless Registration of Point Clouds from TLS
}

\section{and Structured Light Scanner for Heritage Documentation}

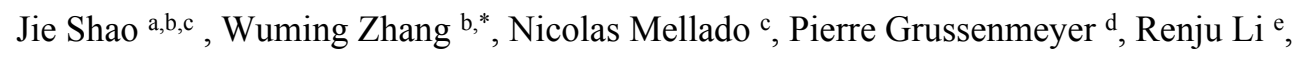 \\ Yiming Chen ${ }^{\mathrm{b}}$, Peng Wan ${ }^{\mathrm{b}}$, Xintong Zhang ${ }^{\mathrm{b}}$, Shangshu Cai ${ }^{\mathrm{b}}$ \\ ${ }^{a}$ Beijing Advanced Innovation Center for Imaging Technology, Capital Normal University, Beijing 100048, China \\ ${ }^{b}$ State Key Laboratory of Remote Sensing Science, Institute of Remote Sensing Science and Engineering, Faculty of \\ Geographical Science, Beijing Normal University, Beijing 100875, China \\ c IRIT, CNRS, University of Toulouse, Toulouse 31062, France \\ d ICube Laboratory, Photogrammetry and Geomatics Group, National Institute of Applied Sciences (INSA), Strasbourg 67084, \\ France

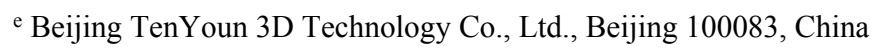

\begin{abstract}
Three-dimensional (3D) model is a major form of cultural heritage documentation. In most cases, the properties of digital artefacts (e.g. readability, coverage) are affected by the acquisition procedure (e.g. device, workflow, conditions) and the characteristics of the physical artefact (e.g. shape, size and materials). In this paper, we study how to combine two acquisition techniques to acquire detailed 3D models of large physical objects. Specifically, we combine two laser scanning instruments: Terrestrial Laser Scanning (TLS) and Structured Light Scanner (SLS). TLS provides millimeter-scale resolution with large field of view, while SLS provides sub-millimeter resolution for limited field of view. This paper focuses on the registration of SLS and TLS point clouds, a critical step which aims at aligning the acquired point clouds in a common frame. Existing registration systems mostly rely on manual post-processing or marker-based alignment. Manual registration is however time consuming and tedious, while markers increase the complexity of scanning and are not always acceptable in cultural site documentation. Therefore, we propose an automated markerless registration and fusion pipeline for point clouds. Firstly, we replace the marker-based coarse alignment by an automated registration of SLS and TLS point clouds; secondly, we refine the alignment of SLS point clouds on TLS data using the Iterative Corresponding Point algorithm; finally, we seamless stitch the SLS and TLS point clouds by globally regularizing the registration error for the all the point clouds at once. Our experiments show the efficiency of the proposed approach on two real-world cases, involving detailed point clouds correctly aligned without requiring markers or manual tuning. This paper provides an operational process reference for automated markerless registration of multi-source point clouds.
\end{abstract}

Keywords: heritage documentation; terrestrial LiDAR; structured light scanner; point clouds registration

\section{Introduction}

The digitalization of Cultural Heritage material is nowadays a common process, enabling long term archiving, simple sharing and digital presentation for research and diffusion to the general public [1]. Due to the rich information usually found in Cultural Heritage materials, e.g. fine and vivid work art, complex and diverse surface, 3D digitalization is today becoming the standard in most application cases, yielding important research efforts for the 3D modeling of Cultural Heritage content. Especially, the choice of a specific acquisition technique is a determining factor for the quality and properties of the virtual description of the Cultural Heritage artefacts. In some cases, it might be necessary to combine multiple acquisition techniques at once [2], which imply a final registration step to merge the heterogeneous data in a unique and consistent

Corresponding author.

E-mail address: wumingz@bnu.edu.cn (Wuming Zhang). 
model.

The simplest form of 3D models is a set of 3D coordinates sampling an object surface, called a point cloud. Most acquisition techniques acquire physical objects as point clouds, e.g., laser scanners, structure from motion and structured light [3]. Laser scanning is nowadays a very common acquisition technique, and we refer the interested reader to the survey by Vacca et al. [4] on laser-based acquisition for Cultural Heritage applications. We consider in this work Terrestrial Laser Scanning (TLS). A TLS instruments can provide high precision 3D point cloud with millimeter accuracy over varying field of views. In practice, devices with large field of view (e.g., 360 degrees in the horizontal direction) have low horizontal sampling rate which reduces the amount of details acquired by the scanner. Structure from motion (SFM) extracts corresponding points from overlapping images and generates detailed 3D models [5], but without guaranteed accuracy and correctness [6]. Meanwhile, in some specific cases with high surface erosion and other defects, the lack of texture information might limit the efficiency of SFM techniques. Instead of relying on the surfaces textures, Structured Light Scanners (SLS) project light patterns on the object and obtains a point cloud model from distortions of the pattern [7-8], reaching submillimeter accuracy [9]. Compared with TLS, SLS can get more detailed 3D point clouds. However, due to their limited field of view, SLS instrument might require multi-station scanning to acquire large-scale objects or scenes.

This motivates the need of $3 \mathrm{D}$ registration techniques, which align several point clouds acquired from different points of view and/or with different instruments. Marker-based registration requires positioning artificial markers in scene, and then using these markers to find correspondences between several scans and align the associated point clouds [10]. In practice, complex scenes might require to set a large number of markers so they are visible in multiple scans. In addition to be time consuming, fixing markers on ancient objects might cause secondary damage to the cultural heritage site, which is obviously undesirable. Instead of using physical markers, several techniques automatically extract geometric features, assuming they will be detected consistently in the input point clouds and used as marker substitutes by the registration algorithms [11-12]. In practice however, descriptor-based approaches might not be robust to variations of noise, details and missing data, i.e. the defects that are usually occurring when processing point clouds generated by multiple acquisition systems [13]. Alternatively, exploration-based registration approaches explore the space of rigid transformations to find the configuration maximizing a given metric (e.g., overlap). The state-of-the-art algorithm is 4-Points Congruent Sets (4PCS) [14-15], which matches almost-planar 4-point basis in two input clouds. Despite its efficiency, this approach might in some cases require a lot of computational time [16]. More recently, the variant Super4PCS [17] greatly increased the computational efficiency of 4PCS by reducing the computational complexity required for the exploration.

We also refer the interested reader to the survey by Gomes et al [18], where the authors review the principal steps and associated challenges related to the acquisition and processing of 3D content for Cultural Heritage documentation. After acquisition and coarse registration, the $3 \mathrm{D}$ point clouds transformations need to be refined to avoid error accumulation occurring when combining multiple pairwise registrations.

\section{Research aim}

In order to obtain robust, accurate and detailed 3D models of cultural heritage sites in a non-destructive way, this work combine the advantages of TLS (e.g., large-scale) and SLS (e.g., accuracy) for 3D modeling of Longmen Grottoes. A TLS point cloud represents the whole cultural heritage site, and each SLS point cloud is a detailed part of it. These point clouds are aligned automatically by Super4PCS, and then pairwise registration and global regularization are applied to the point clouds to generate the final 3D models. The main contributions of this work is a practical system for the automated registration of multiple point clouds with 
varying accuracy and sampling, allowing large scale acquisition of Cultural Heritage sites with locally detailed areas.

The rest of this paper is structured as follows: Section 3 describes the experimental material and the registration method of point clouds. The experimental results are presented and discussed in Section 4. Finally, the conclusions are stated in Section 5.

\section{Material and methods}

\subsection{Data description}

Excavation of Longmen Grottoes (Fig. 1) has been ongoing since 493 AD. It is one of three notable stone carving treasures in China and is known as one of the world's greatest classical art treasures. The site was placed upon the UNESCO World Heritage List in 2000, and the World Heritage Committee evaluated that Longmen Grottoes and Buddha is the largest and the most outstanding plastic arts from the later period of Northern Wei Dynasty to the Tang dynasty. These art pieces, which describe religious subjects of Buddhism in detail, represent the peak of Chinese art [19]. The protection of Longmen Grottoes has great significance for Chinese civilization and human art, and motivates its documentation and archiving.

In the past, manual measurement was the main way of cultural heritage information acquisition. In recent years, with the development of computer technology, photogrammetry, LiDAR, computer graphics and so on, protection of cultural heritage has progressed speedily, and displayed in the form of 3D model. 3D model has been widely applied in cultural heritage sites of the world by rich spatial information and the development of 3D information acquisition sensors [20-21]. However, because of long-term sunlight exposure, rain erosion and weathering factors, Longmen Grottoes statues have become so fragile that collapses occurred at any time. Therefore, high accuracy and non-destructive acquisition of 3D models is urgent for protection of Longmen Grottoes at present. Due to the fine and detailed structure information of Longmen Grottoes, the resolution of TLS is not enough to meet the demand; SLS has a higher resolution, but its field of view is limited, and multiple SLS point clouds registration easily produces cumulative error. Therefore, this paper combined the advantages of SLS and TLS to obtain 3D surface information of Longmen Grottoes statue.

The device parameters of SLS and TLS are shown in Table 1. For our experiments, we focused on one statue as experimental sample, and the height of the statue is nearly $0.6 \mathrm{~m}$, the average width of the statue is nearly $0.3 \mathrm{~m}$. 1 TLS point cloud and some SLS point clouds are acquired as follows (Fig. 2).

\subsection{Method}

In order to perform automated markerless registration of point clouds from TLS and SLS for heritage documentation, this paper studies the registration from three aspects: pairwise coarse and fine registration, and global regularization. Coarse registration processes pairs of point clouds to align them roughly; fine registration refines the rough transformation estimate; global regularization removes the cumulative error introduced when combining multiple pairwise registrations. The workflow is as shown in Fig. 3.

\subsubsection{Coarse Pairwise Registration}

The goal of the coarse pairwise registration step is to find the relative position of each SLS point cloud on the TLS point cloud. As previously stated, marker-based alignment can provide such rough estimate, however it increases the acquisition time and may damage the cultural heritage artefacts. Alternatively, we propose in this work to use the state of the art registration technique Super4PCS, an algorithm which computes the rough alignment between one source point cloud to a target point cloud. In order to align all SLS point clouds on the same reference frame, we sequentially register each SLS point clouds on the TLS point cloud. 
Super4PCS estimates a coarse alignment between two point clouds by exploring the set of rigid transformations (rotations and translations) using the input point clouds as priors. More formally, it selects 4 almost coplanar points in the target point cloud to form a so-called 4-point basis. Then it looks for all 4-points configurations with similar geometric properties in the source point cloud. For each configuration, a geometric transformation candidate $T_{i}$ is computed by aligning the 4-points to the basis in the target cloud. Super4PCS selects the transformation that maximizes the overlap between the source and target clouds. The input point clouds are locally overlapping if their distance is locally less than a given threshold. Considering that the TLS point cloud has been acquired with 5 millimeters precision, we set the overlapping distance threshold at 1 centimeter for all our experiments. We used the implementation provided by the OpenGR library [22] for our experiments (Fig. 4 (a)).

Fig. 4 (a) shows two results of coarse alignment between a SLS point cloud (white) and the TLS point cloud (red). Super4PCS outputs correct rough estimate of the transformations, i.e. the SLS point clouds are overall well positioned on the TLS point cloud, but still need to be further refined in a second step.

\subsubsection{Fine Pairwise Registration}

The goal of the fine pairwise registration step is to slightly move the SLS point clouds so they fit as much as possible the TLS point cloud. This is a very standard process in point cloud processing, also known as local pairwise registration. The reference algorithm to solve this problem is the Iterative Closest Point (ICP) algorithm that was proposed by Besl and Mckay [23]. ICP is an iterative algorithm that minimizes the distance between pairs or corresponding points in the two point clouds. The algorithm repeats the selection of corresponding points to compute an optimal rigid-body transformation until converging on accurate matching results. Several variants have been proposed under the name Iterative Corresponding Point (see recent survey by Pomerleau et al. [24]), and ICP is nowadays considered as a very mature algorithm, implemented in most 3D software.

In a nutshell, given a source point cloud (in our case, any SLS cloud) and a target point cloud (in our case, the TLS cloud), the ICP algorithm first establishes a set of pair-correspondences between points in two clouds. Then, it computes a 3D rigid-body transformation minimizing a given error metric between the corresponding points, e.g. the point-to-plane distance.

To be consistent with the parameters used during the coarse registration step, we set the ICP error threshold at $1 \mathrm{~cm}$, the maximum number of iteration to 20 , and the convergence threshold $\eta=1.0^{-5}$. We show in Fig. 4 (b) how ICP refines the transformations (we used CloudCompare [25] for our experiments).

In this example, ICP produces good results even if it has to solve a relatively large motion between the rough estimate and the target configuration (Fig. 4 (b)). As registration is performed only between each SLS point cloud and the TLS point cloud, there is no guaranty that two overlapping SLS point cloud will be well aligned by this process (in blue boxes of Fig.4 (b)). This motivates the need of final global regularization to minimize the registration error globally between all point clouds.

\subsubsection{Global regularization}

In order to ensure gapless registration, we propose to globally adjust the sequence of SLS point clouds and TLS point cloud with a large overlap. As some point clouds may overlap, and other not, we propose to build a pose graph (Fig. 5) as follow: each point cloud is a vertex in the graph (denoted TLS $v_{0}$ or SLSv $v_{i}$ ), and two vertices are connected by an edge $e_{i}$ if the associated point clouds are overlapping. As for previous stages, we consider that two clouds are overlapping if their local distance is less than 1 centimeter, and we request at least $30 \%$ overlap to create an edge. As the SLS point clouds have been registered to the source TLS point cloud in previous steps, it is very likely that all SLS $v_{i}$ vertices are connected to TLS $v_{0}$ in the graph. 
Once the graph is created, we use multi-view ICP [26], an extension of the ICP algorithm that minimizes the registration error between multiple point clouds at once. It follows the overall ICP algorithm, except that the error is computed for all pairs of points between all connected clouds. It outputs the set of transformations for all the movable vertices in the pose graph. In our case, all SLS vertices $v_{i}$ are marked as movable, but not the $\mathrm{TLS} v_{0}$, which defined the common registration basis for the whole dataset. We used the implementation by Glira et al [27], and use same stopping criterions as for fine pairwise registration. The result of global regularization is shown as Fig. 6.

\section{Results and discussion}

We evaluated our approach on two datasets, acquired on two different Buddha in Longmen Grottoes (see statistics in Table 2). Each dataset is composed by one reference TLS point cloud spanning the whole scene, and multiple SLS point clouds detailing parts of the scene. The variations of sampling and amount of details between the SLS and TLS point clouds are shown in Fig. 7.

In this section, we evaluate each step of our approach on scene 2 that we believe challenging, considering:

1) The symmetry of the scene, which may introduce ambiguities at the coarse registration step,

2) The large feature-less regions that prevent to use descriptor-based alignment, and,

3) The variation of sampling and level of details between the SLS and TLS point clouds.

We also demonstrate in supplementary materials how our approach performs on scene 2 . All our experiments have been performed on an Intel Xeon E5-2640 2.40GHz with 40 cores. We emphasis that both the coarse and fine registration steps can be ran in parallel, in order to align each SLS point cloud to the TLS reference point cloud simultaneously. We used single thread Matlab implementation [29] for our experiments, and better performances can be expected with optimized code. For our experiments we stop the ICP iterations when the registration error increases. As shown in Table 2, computing the coarse alignment between all clouds in parallel takes $2 \mathrm{~m} 15 \mathrm{~s}$ with our setup, for $91 \mathrm{~m} 30$ s of total processing time. In addition, TLS point cloud and all SLS point clouds of all scenes were re-sampled according to the voxel size of $1 \mathrm{~mm}$ for the global regularization.

\subsection{Coarse and fine pairwise registration}

To evaluate the robustness of the coarse registration step, we used a fixed set of parameters for the whole dataset: target overlap 90\%, registration tolerance $5 \mathrm{~mm}$, and 2500 samples per point cloud. Registration results are shown in Fig. 8. 39 SLS point clouds were correctly registered (in white, Fig. 8(a)), and 2 SLS point clouds were not correctly registered (in yellow, Fig. 8 (b-c)).

As shown in Fig. 8 (c), the two SLS point clouds describe mostly flat surfaces with low but regular curvature. The Super4PCS algorithm finds the transformation that maximizes the overlap between the two input clouds given the input tolerance value. In these two cases, it appears that this metric is not enough to distinguish between the correct pose and the found pose, where the SLS point cloud spans a part of the TLS point cloud. In practice, these two failure cases might be corrected by adjusting the Super4PCS parameters; we obtained good results by reducing the target overlap to $80 \%$.

In summary, except for two features-less point clouds, all of the SLS point clouds can be registered with the TLS point cloud, which shows the high reliability of the Super4PCS algorithm for coarse registration. Also, in all cases we could refine the estimated transformation using ICP (see Section 3.2.2).

\subsection{Global regularization}

During the coarse alignment and fine registration steps, each SLS point cloud is registered to the reference TLS point cloud, without considering the registration error between adjacent SLS point clouds. In practice, 
these gaps do not meet the standard of heritage documentation, and a global regularization is required.

In this section we evaluate the influence of the TLS point cloud in the global regularization step. In Fig. 9(a), we show results of scene 1, where only SLS point clouds are considered in the global regularization graph. The goal of this experiment is to simulate the registration of detailed point clouds without using a reference point cloud. Unsurprisingly, the SLS point clouds are correctly aligned, but the surface they describe deviates from the TLS point cloud: registration error is accumulated and the overall registration quality is reduced even if the registration error remains low.

On the other hand, we show in Fig. 9 (b) how using the TLS point cloud as reference greatly improves the registration result. By combining SLS-SLS and SLS-TLS registration at once, our approach provides point clouds with low local and global registration error.

\section{Conclusions}

The paper researched an automated markerless registration of SLS point clouds and TLS point cloud for the heritage documentation of Longmen Grottoes Buddha from three aspects: coarse alignment, pairwise registration, and global regularization. Our experiments showed that the SLS clouds can represent accurate surface information of Buddha, but may lead to high accumulative error due to the registration process. On the other hand, the TLS could get a whole 3D model of Buddha with one scanning, but the surface information is not detailed enough. The combination of SLS and TLS could not only represent details for the whole Buddha site, but also remove the accumulative error of a sequence of SLS point clouds registration. Besides, automated markerless registration approach reduced dependence on artificial markers and manual work, which improved the work efficiency. The proposed method provides an operational pipeline reference for automated markerless registration of multi-source point clouds. All our experiments were performed using available processing tools, and we will provide macros and scripts as reference for workers of digital documentation of heritage and point cloud processing.

The main aim of this research is to explore an automated markerless registration approach of point clouds for heritage documentation. The first step of our pipeline, i.e., coarse alignment, is a key factor in automated registration of point clouds. In case of feature less surfaces, it might however fail in some cases and becomes the main limitation for the high success rate of registration. In future work, we will focus on reducing the sensitivity of parameters which influence the success rate of coarse alignment and thus improving the success rate of the overall pipeline.

\section{Acknowledgements}

This work was supported by the National Key Research and Development Program of China Grant No. (NO.2016YFB0501404) and the National Natural Science Foundation of China Grant Nos. 41671414, 41331171 and 41171265 . This work was also supported by the Open Research Fund of Key Laboratory of Digital Earth Science, Institute of Remote Sensing and Digital Earth, Chinese Academy of Sciences Grant No. 2014 LDE015.

\section{References}

[1] F. Remondino, A. Rizzi, Reality-based 3D documentation of natural and cultural heritage sites-techniques, problems, and examples, Applied Geomatics, 2010, pp. 85-100.

[2] W. Zhang, C. Wang, X. Xi, 3D scan of ornamental column (huabiao) using terrestrial lidar and hand-held imager, ISPRS, International Archives of the photogrammetry, Remote Sensing and Spatial Information Sciences, XL-5/W7(2015) 491494. 
[3] C.G. Serna, R. Pillay, A. Trémeau, Data fusion of objects using techniques such as laser scanning, structured light and photogrammetry for cultural heritage applications, Lecture Notes in Computer Science - Computational Color Imaging, 2015, pp. 208-224.

[4] G. Vacca, M. Deidda, A. Dessi, M. Marras, Laser scanner survey to cultural heritage conservation and restoration, ISPRS International Archives of the Photogrammetry, Remote Sensing and Spatial Information Sciences, XXXIX-B5(2012) 589594.

[5] J. McCarthy, Multi-image photogrammetry as a practical tool for cultural heritage survey and community engagement. J. Archaeol. Sci. 43(2014) 175-185.

[6] C. Santagati, L. Inzerillo, F.D. Paola, Image-based modeling techniques for architectural heritage 3d digitalization: limits and potentialities, The International Archives of the Photogrammetry, Remote Sensing and Spatial Information Sciences, Volume XL-5/W2(2013) 550-560.

[7] J.W., Liu, Z.Q. Jiang, X. Sun, H. Hu, Integration of close range photogrammetry and structured light scanner for cultural heritage documentation, Advanced Materials Research, 2012, pp. 1966-1969.

[8] D. Akca, 3D modeling of cultural heritage objects with a structured light system, Mediterranean Archaeology \& Archaeometry, 12(2012) 139-152.

[9] F. Buchónmoragues, J.M. Bravo, M. Ferri, J.V. Sánchezpérez, Application of structured light system technique for authentication of wooden panel paintings, Sensors. 16(2016) 881-890.

[10] T. Hilker, N.C. Coops, D.S. Culvenor, G. Newnham, M.A. Wulder, C.W. Bater, A. Siggins, A simple technique for coregistration of terrestrial LiDAR observations for forestry applications, Remote Sens. Lett. 3(2012) 239-247.

[11] P.M. Segundo, L. Gomes, O.R.P. Bellon, L. Silva, Automating 3D reconstruction pipeline by surf-based alignment, IEEE International Conference on Image Processing, 2012, pp. 1761-1764.

[12] G. Bitelli, G. Castellazzi, A.M. D’Altri, S.D. Miranda, A. Lambertini, I. Selvaggi, Automated voxel model from point clouds for structural analysis of cultural heritage, The International Archives of the Photogrammetry, Remote Sensing and Spatial Information Sciences, XLI-B5(2016) 191-197.

[13] N. Mellado, M. Dellepiane, R. Scopigno, Relative scale estimation and 3D registration of multi-modal geometry using Growing Least Squares, IEEE Transactions on Visualization and Computer Graphics, 22(2016) 2160-2173.

[14] D. Aiger, N.J. Mitra, D. Cohen-Or, 4-points congruent sets for robust pairwise surface registration, ACM Trans. Graph. 27(2008) 1-10.

[15] P.W. Theiler, J.D. Wegner, K. Schindler, Markerless point cloud registration with keypoint-based 4-points congruent sets, ISPRS Annals of the Photogrammetry, Remote Sensing and Spatial Information Sciences, Vol. II-5/W2(2013) 283-288.

[16] P.W. Theiler, J.D. Wegner, K. Schindler, Keypoint-based 4-points congruent sets - automated marker-less registration of laser scans, ISPRS-J. Photogramm. Remote Sens. 96(2014) 149-163.

[17] N. Mellado, D. Aiger, N.J. Mitra, SUPER 4PCS fast global pointcloud registration via smart indexing, Comput. Graph. Forum, 33(2014) 205-215.

[18] L. Gomes, O.R.P. Bellon, L. Silva, 3D reconstruction methods for digital preservation of cultural heritage: A survey, Pattern Recognit. Lett. 50(2014) 3-14.

[19] D. Wang, Internationalizing heritage: UNESCO and China's Longmen Grottoes, China Information, 24(2010) 123-147.

[20] O. Chee, C. Siew, Z. Majid, H. Setan, 3D documentation and preservation of historical monument using terrestrial laser scanning, Geoinformation Science Journal, 10(2010) 73-90.

[21] M.L. Brutto, M. Spera, Image-based and range-based 3d modelling of archaeological cultural heritage: the Telamon of Temple of Olympian ZEUS in Agrigento (Italy), ISPRS - International Archives of the Photogrammetry, Remote Sensing and Spatial Information Sciences, 16(2012) 515-522.

[22] OpenGR:A C++ library for 3D Global Registration, N. Mellado and others, Accessed May 2018.

[23] P.J. Besl, N.D. Mckay, A method for registration of 3-D shapes, IEEE T. Pattern Anal. 14(1992) 239-256. 
[24] F. Pomerleau, F. Colas, R. Siegwart, A Review of Point Cloud Registration Algorithms for Mobile Robotics. Found. Trends Robot, 4(2015) 1-104.

[25] CloudCompare. 3

3D (http://www.cloudcompare.org/doc/wiki/index.php?title=ICP. Accessed 6 October 2015).

\section{5}

[26] K. Pulli, Multiview registration for large data sets, In Proceedings of the 2nd international conference on 3-D digital imaging and modeling (3DIM’99), IEEE Computer Society, Washington, DC, USA, 1999, 160-168.

[27] P. Glira, N. Pfeifer, C. Ressl, C. Briese, A correspondence framework for ALS strip adjustments based on variants of the ICP algorithm, Journal for Photogrammetry, Remote Sensing and Geoinformation Science, 2015, pp. 275-289.

[28] R. Vergne, R. Pacanowski, P. Barla, X. Granier, C. Schlick, Radiance Scaling for Versatile Surface Enhancement, I3D 10: Proc. symposium on Interactive 3D graphics and games, Feb 2010, Boston, United States. ACM, 2010.

[29] GlobalICP Matlab scripts, Philipp Glira, Accessed May 2018. 


\section{Figure captions}

303 Fig.1. Longmen Grottoes.

304 Longmen Grottoes is one of three notable stone carving treasures in China and is known as one of the world's greatest classical

305 art treasures. The World Heritage Committee evaluated that Longmen Grottoes and Buddha is the largest and the most outstanding plastic arts from the later period of Northern Wei Dynasty to the Tang dynasty.

Fig. 2. Point cloud of Longmen Grottoes statue.

308 (a) shows two SLS point clouds of a statue; (b) shows one TLS point cloud of a statue.

309 Fig. 3. The registration pipeline and overview.

310 (a) shows the schematic view of the registration pipeline; (b) shows the overview of the registration pipeline from a data 311 perspective, which includes four steps: (1) input, (2) coarse registration, (3) fine registration, and (4) global registration. The

312

313

314

315

316

317

318

319 input consists of two types of point clouds: one sparse (TLS, in orange) and multiple dense (SLS). After fine registration between each SLS point clouds and the TLS reference point clouds, misalignments between SLS point clouds (black circle in the figure of fine registration) are solved by the process of global registration.

Fig. 4. Results of Super4PCS and fine pairwise registration.

(a) shows the result of Super4PCS, which estimates the transformation between each SLS point clouds (white) and the TLS point cloud (red); (b) shows the result after fine pairwise registration, where the transformations are refined with ICP. Note that the overlapping parts (blue box) may not be properly aligned as the error between the SLS point clouds is not considered for these two steps.

Fig. 5. Pose graph of global regularization.

SLS point clouds $\left\{\mathrm{v}_{1}, \mathrm{v}_{2}, \mathrm{v}_{3} \ldots \mathrm{v}_{\mathrm{n}}\right\}$ are overlapping with the TLS point cloud and thus connected to $\mathrm{v}_{0}$. Also, all pairs of SLS point clouds with at least $30 \%$ overlap are connected in the graph.

Fig. 6. Global regularization results.

(a) shows the result of 16 SLS point clouds registration; (b) shows the TLS point cloud; (c) shows TLS and SLS point clouds in the same frame.

Fig. 7. The variations of details between SLS and TLS point cloud.

SLS point cloud (a) and TLS point cloud (b) from scene 1 rendered with Radiance Scaling [28].

Fig. 8. Results of coarse alignment.

(a) shows correct coarse alignment between each SLS point cloud and TLS point cloud; (b) shows incorrect coarse alignment;

(c) shows the 2 incorrect SLS point clouds.

Fig. 9. Results of global regularization.

(a) shows the global regularization result of only SLS point clouds, (a-1) and (a-2) show the horizontal and vertical cross section of point cloud in yellow box, respectively; (b) shows the global regularization result combining TLS with SLS point clouds, (b1) and (b-2) show the horizontal and vertical cross section of point cloud in yellow box, respectively. (TLS in red; SLS in white) 
Tables

336 Table 1

337 Device parameters

\begin{tabular}{ccc}
\hline Parameters & SLS & TLS \\
\hline Model & OKIO-5M-400 & RIEGL VZ-1000 \\
Max measure distance & $1.5 \mathrm{~m}$ & $1,200 \mathrm{~m}$ \\
Field of view & $0.4 \mathrm{~m} \times 0.3 \mathrm{~m}($ at max distance $)$ & $100^{\circ} \times 360^{\circ}$ \\
Measure precision & $0.015 \mathrm{~mm}$ & $5 \mathrm{~mm}$ \\
\hline
\end{tabular}

\section{Table 2}

Datasets properties and processing time. For the coarse alignment and fine registration steps, we detail for each scene the processing time needed to register the SLS point cloud on the TLS point cloud in parallel, and the total time when registering the SLS point clouds sequentially on a single processing unit.

\begin{tabular}{|c|c|c|c|c|c|c|}
\hline & \multicolumn{2}{|c|}{ SLS point clouds } & \multirow{2}{*}{$\begin{array}{c}\text { TLS point cloud } \\
\text { \#vertices }\end{array}$} & \multicolumn{3}{|c|}{ Processing time - pairwise (total) } \\
\hline & \#clouds & $\begin{array}{c}\text { \#vertices/clou } \\
\text { d }\end{array}$ & & Coarse registration & Fine registration & $\begin{array}{c}\text { Global } \\
\text { regularization }\end{array}$ \\
\hline Scene 1 & 16 & 859,774 & $2,777,511$ & $1 \mathrm{~m} 10 \mathrm{~s}(18 \mathrm{~m} 40 \mathrm{~s})$ & $3.1 \mathrm{~s}(50 \mathrm{~s})$ & $12.7 \mathrm{~m}$ \\
\hline Scene 2 & 41 & 926,830 & $2,732,000$ & $2 \mathrm{~m} 15 \mathrm{~s}(91 \mathrm{~m} 30 \mathrm{~s})$ & $4.8 \mathrm{~s}(3 \mathrm{~m} 20 \mathrm{~s})$ & $27.5 \mathrm{~m}$ \\
\hline
\end{tabular}




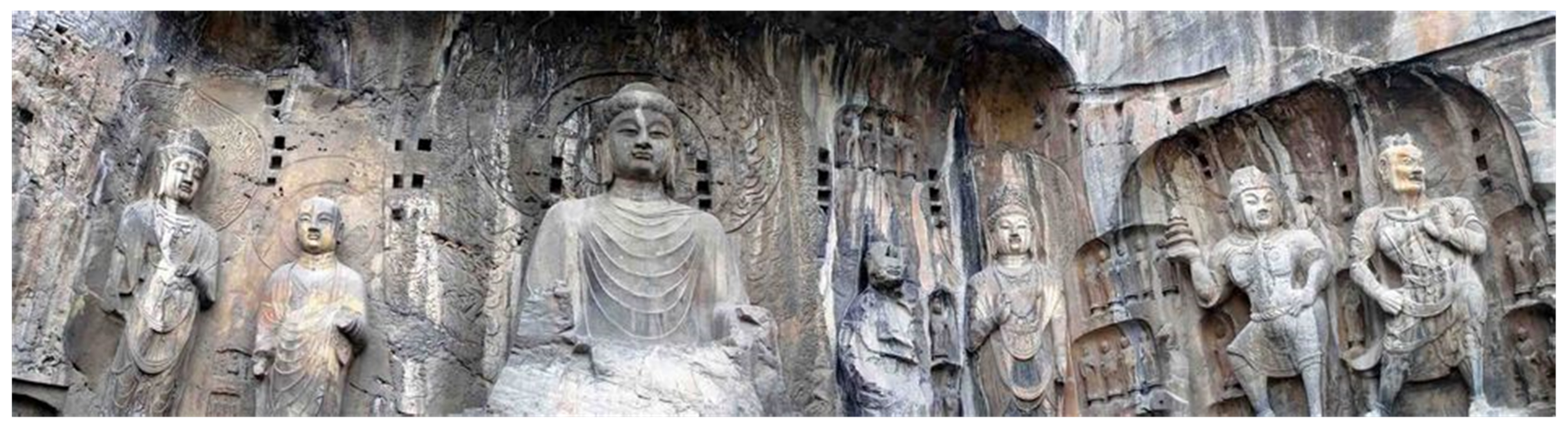





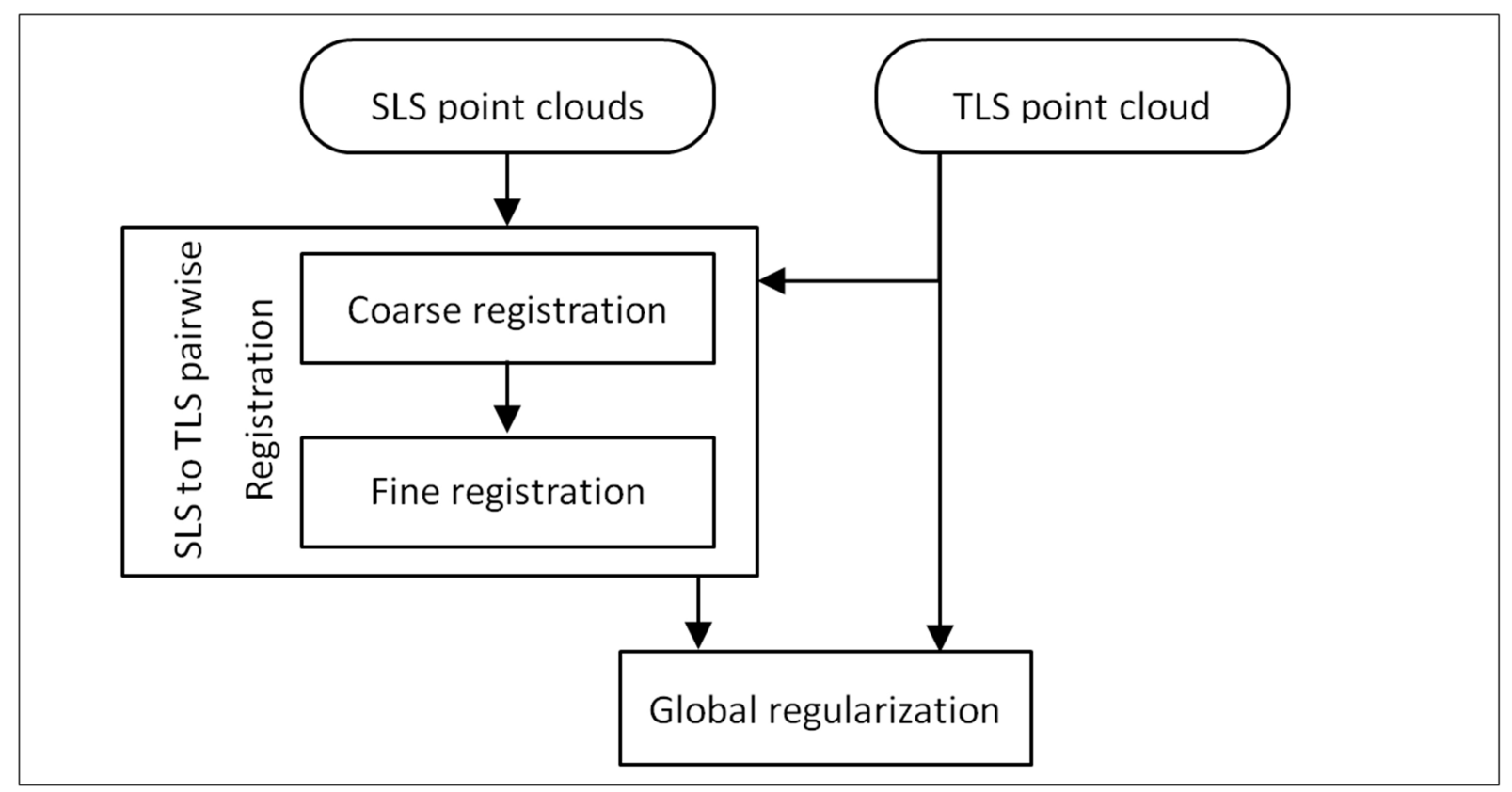

(a)

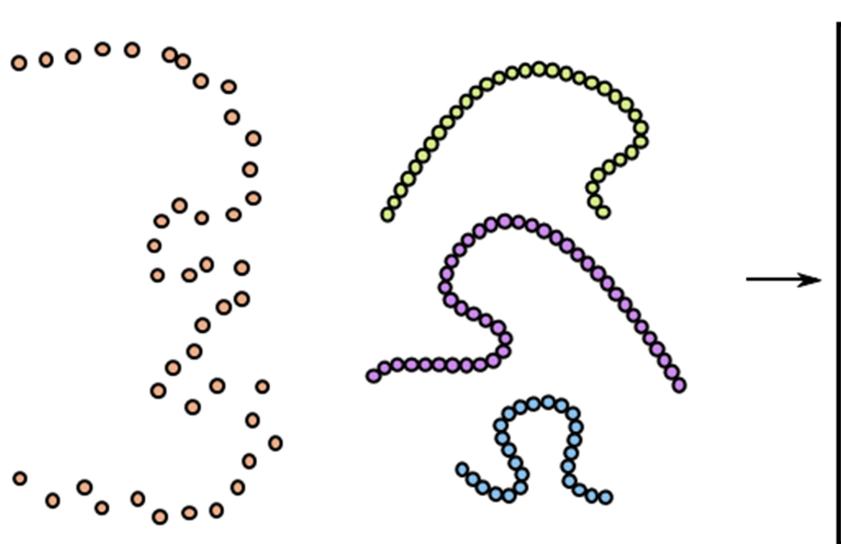

(1)

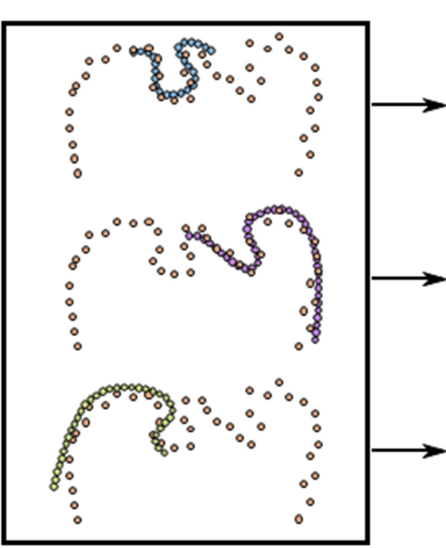

(2)

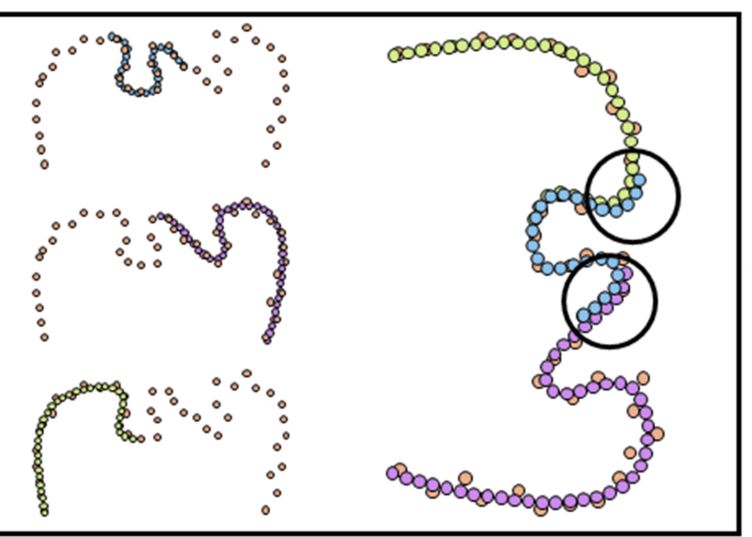

(3)

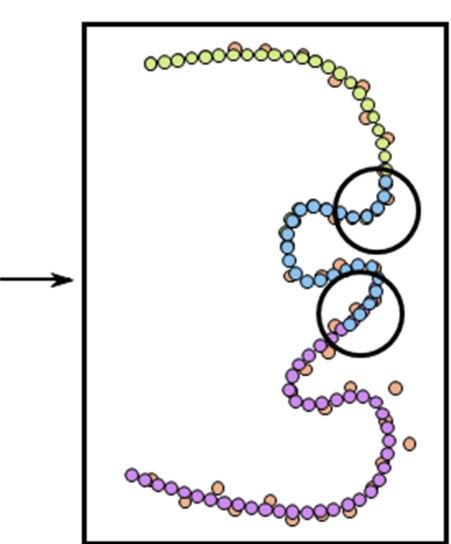

(4)

(b) 


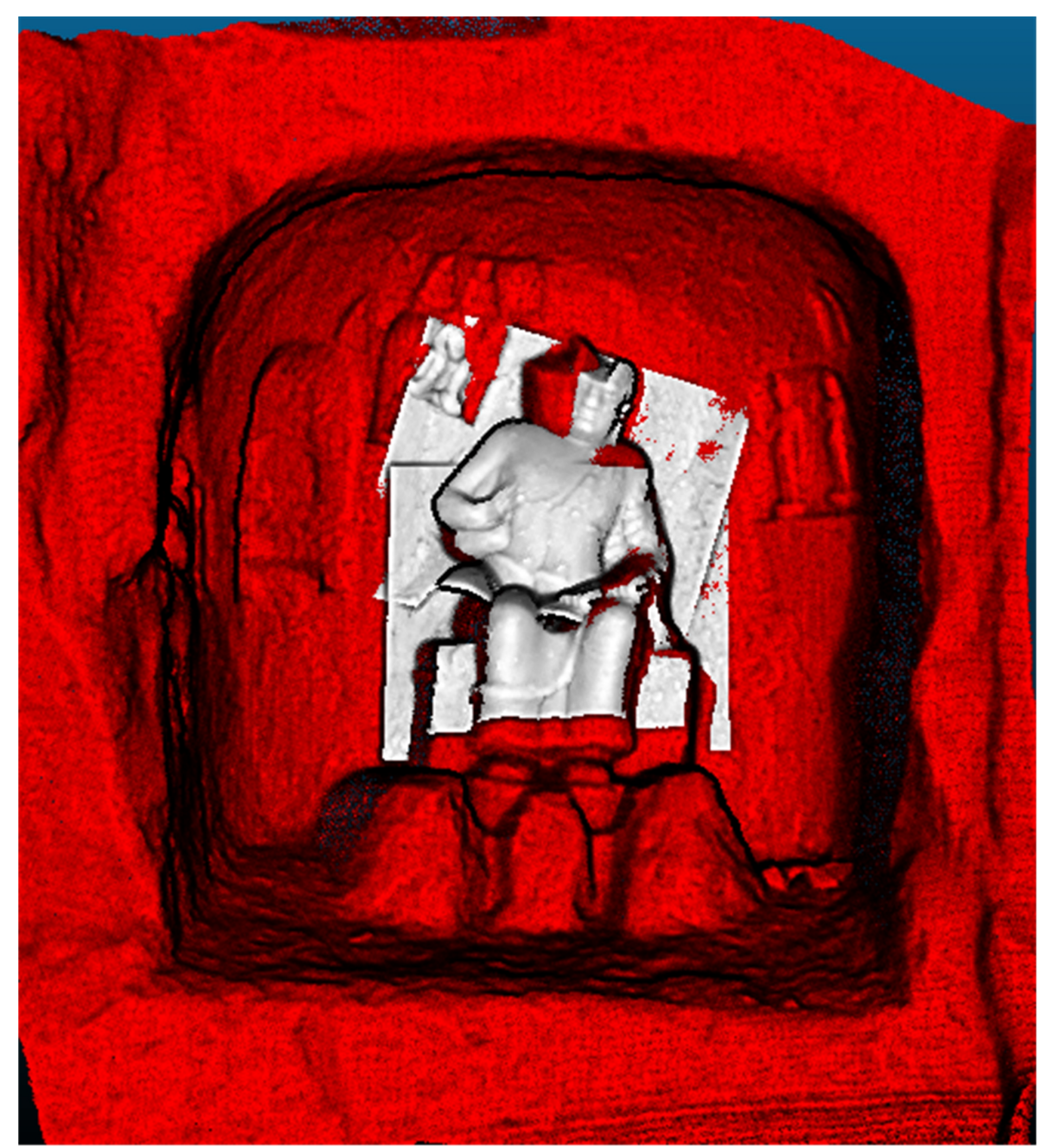

(a)

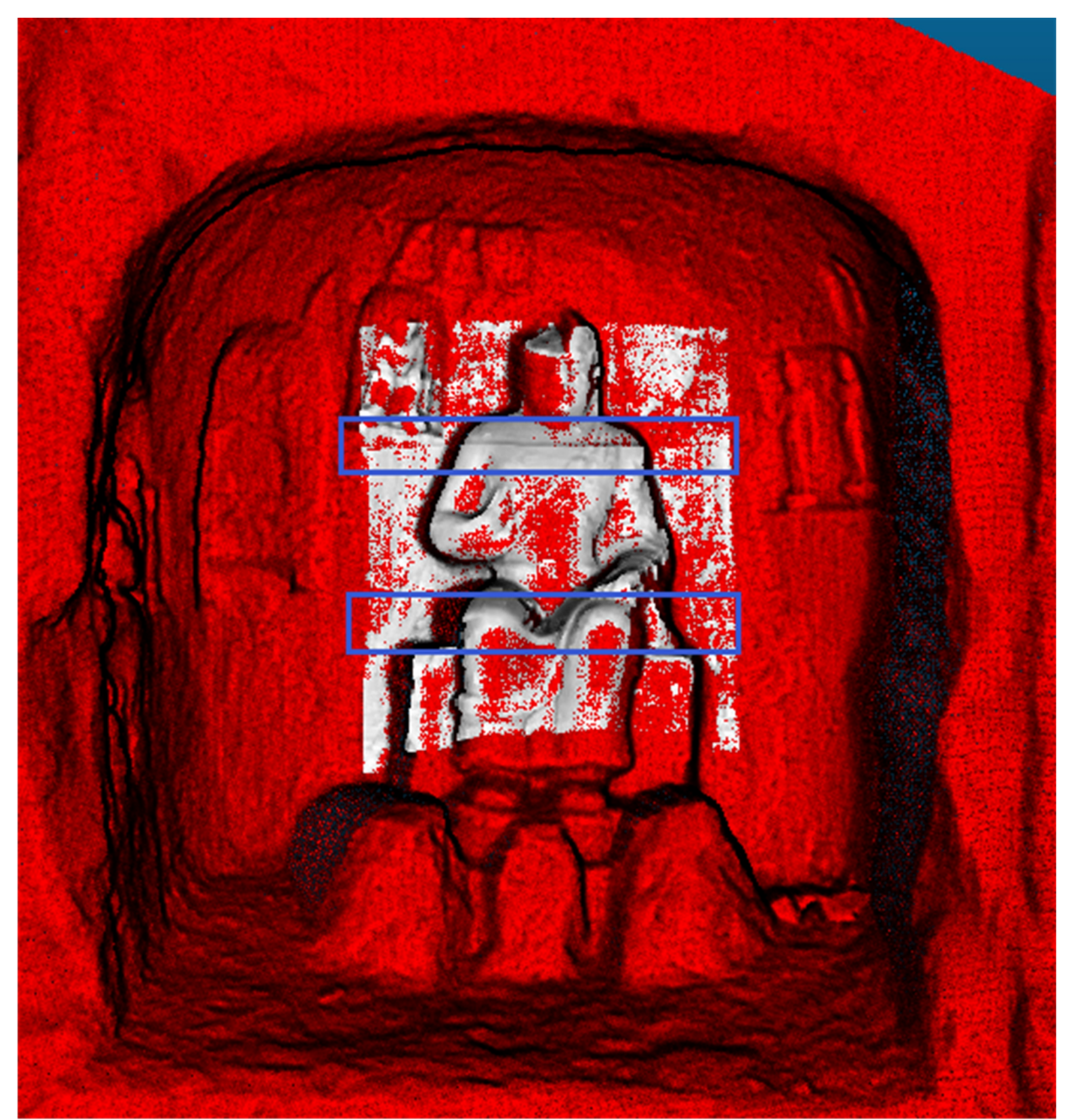

(b) 


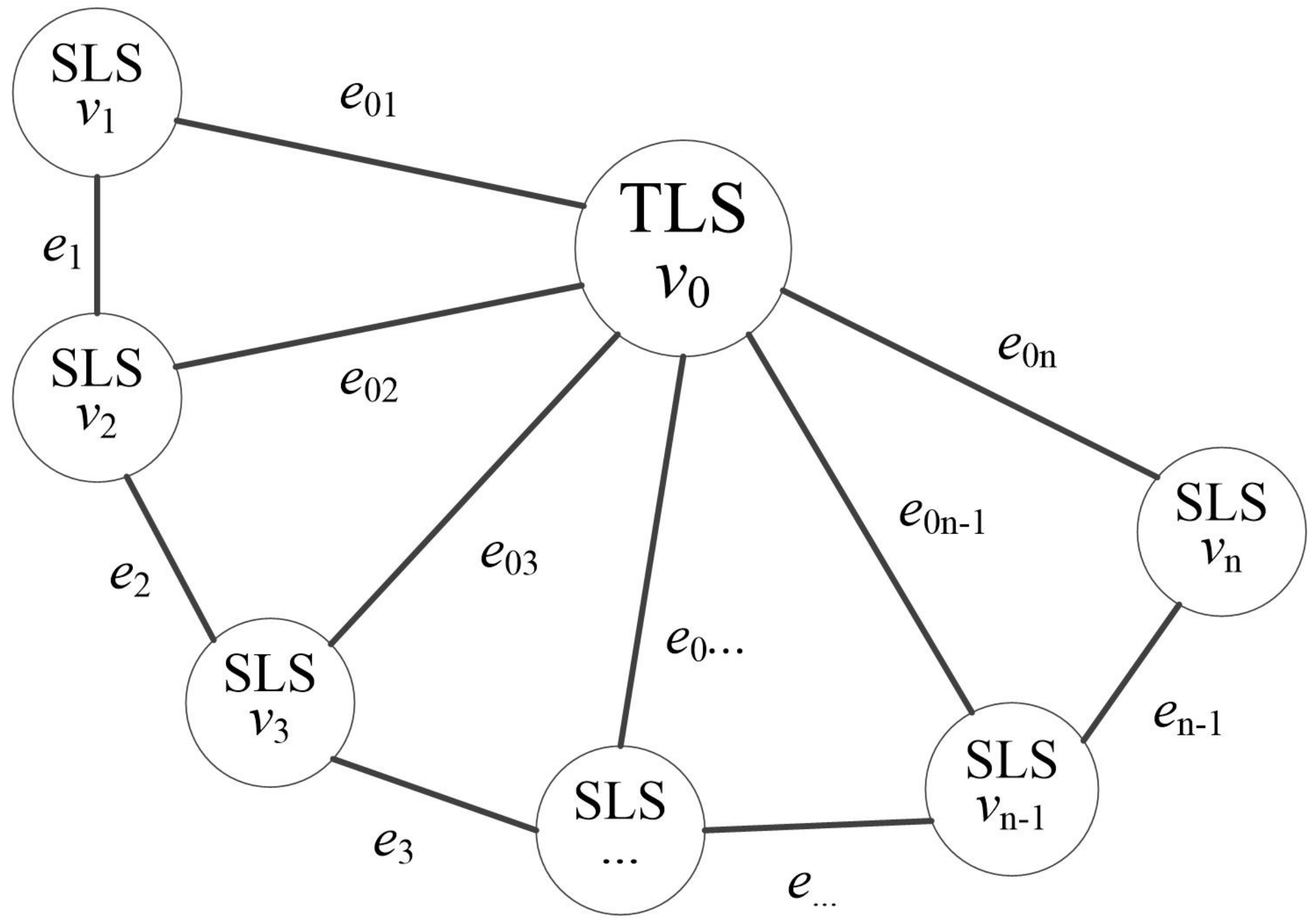



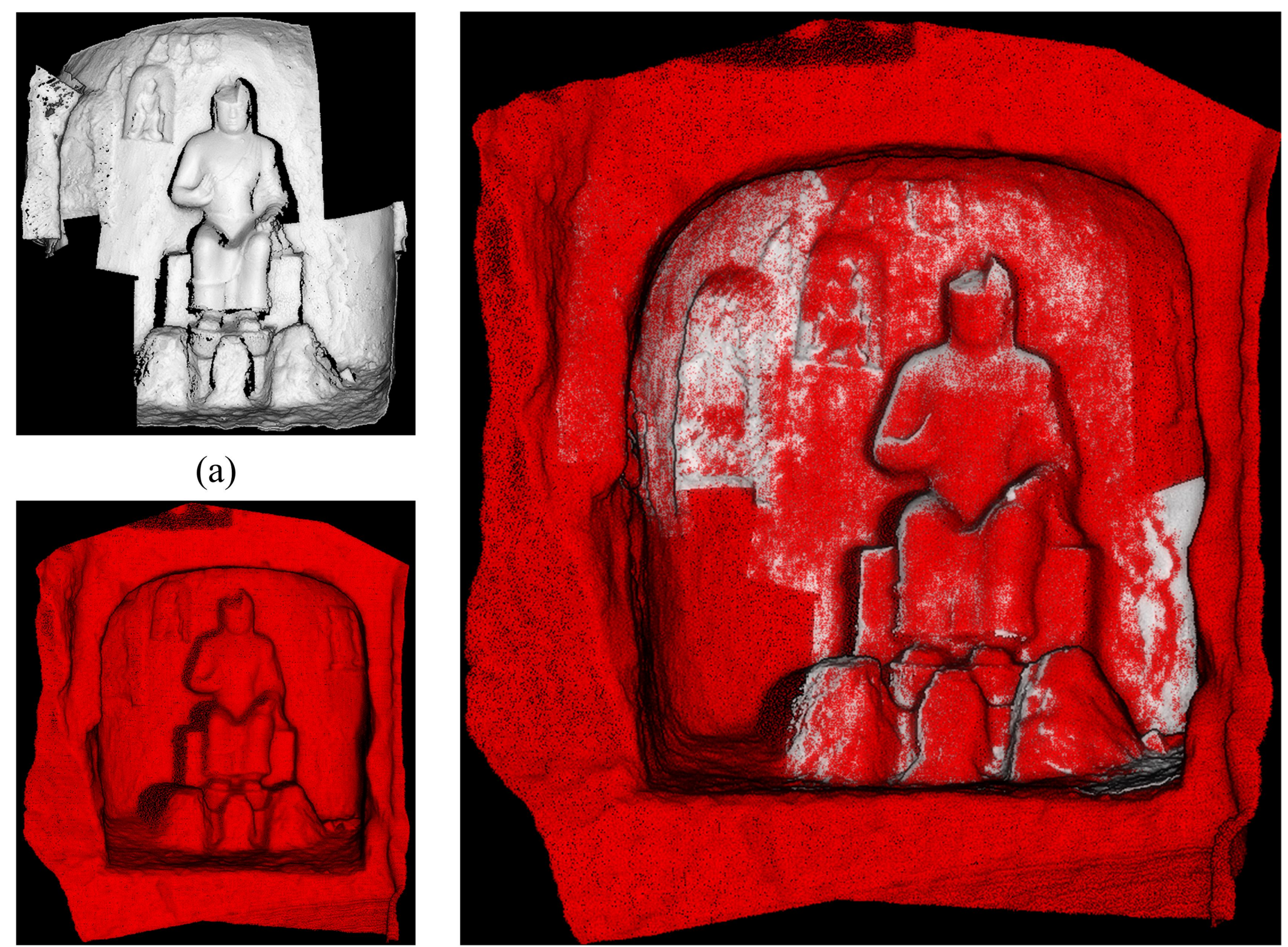

(b)

(c) 

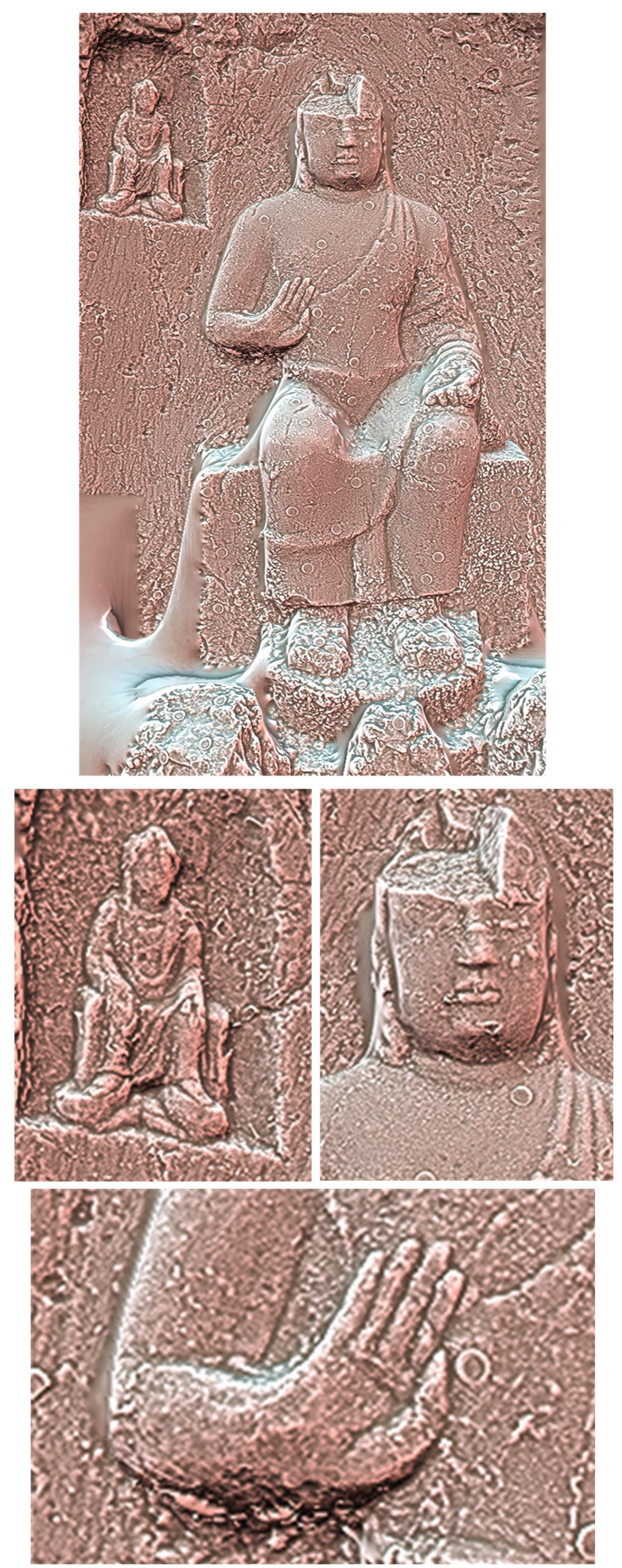

(a)

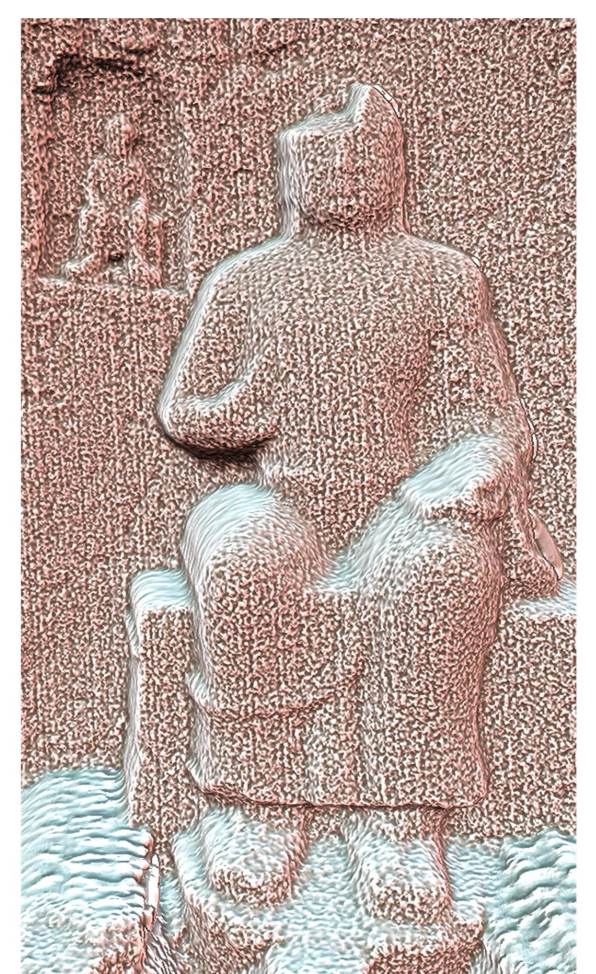

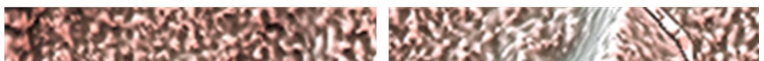
15. 30 .

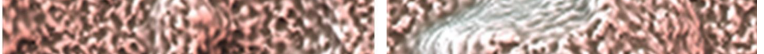
f 17. s

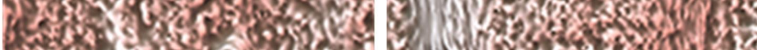
1.6. 3.

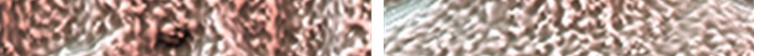
4 2 .r.

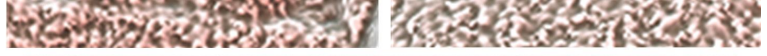

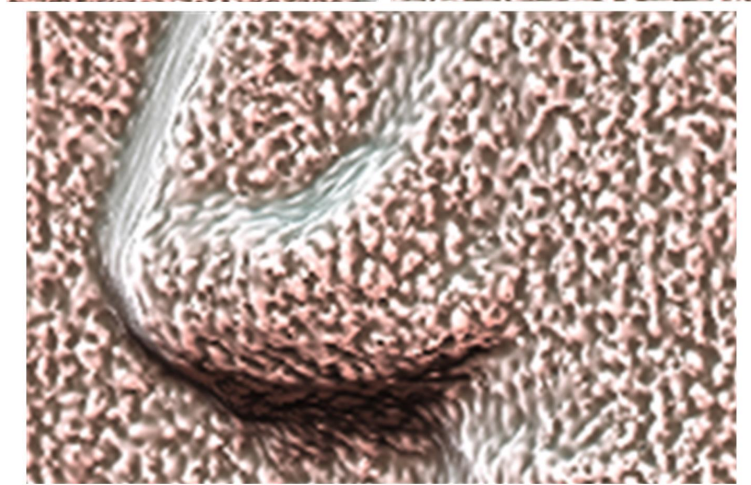

(b) 


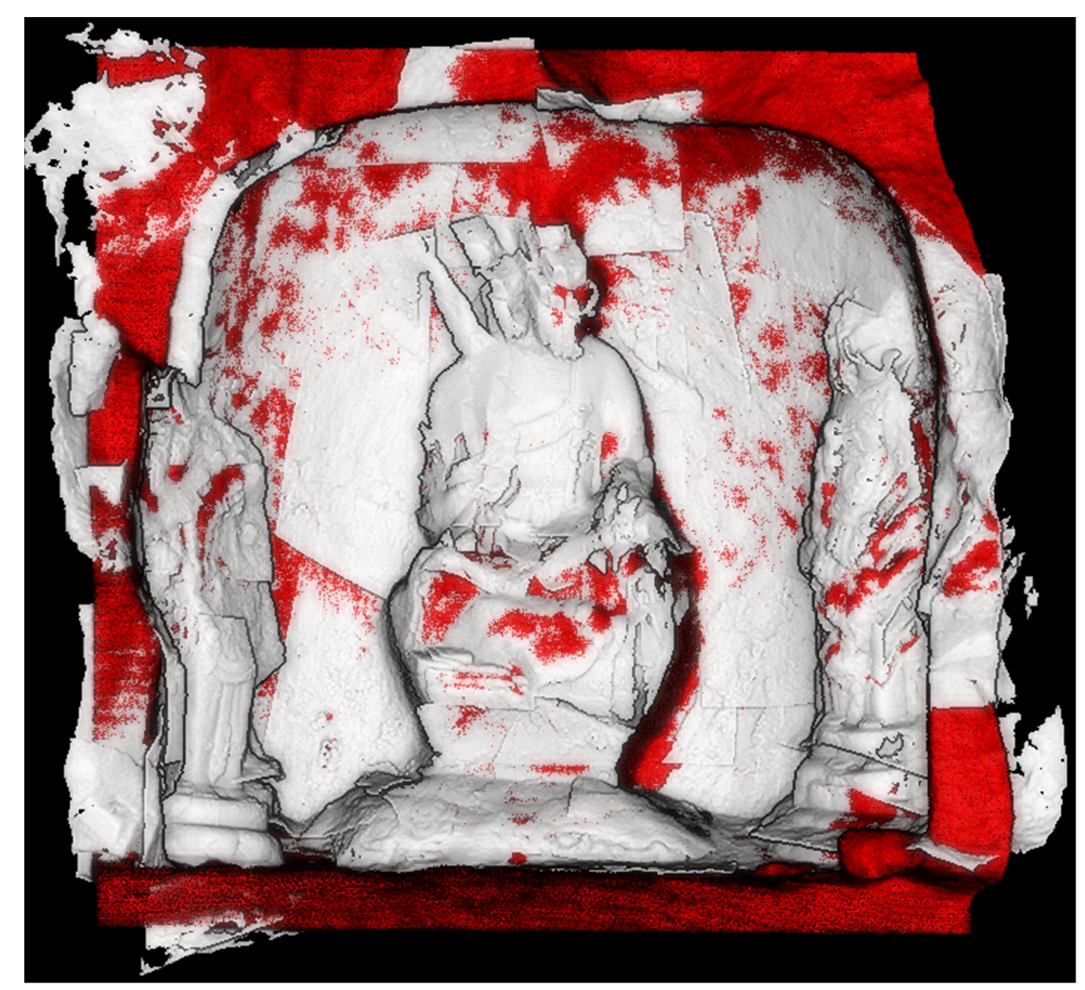

(a)

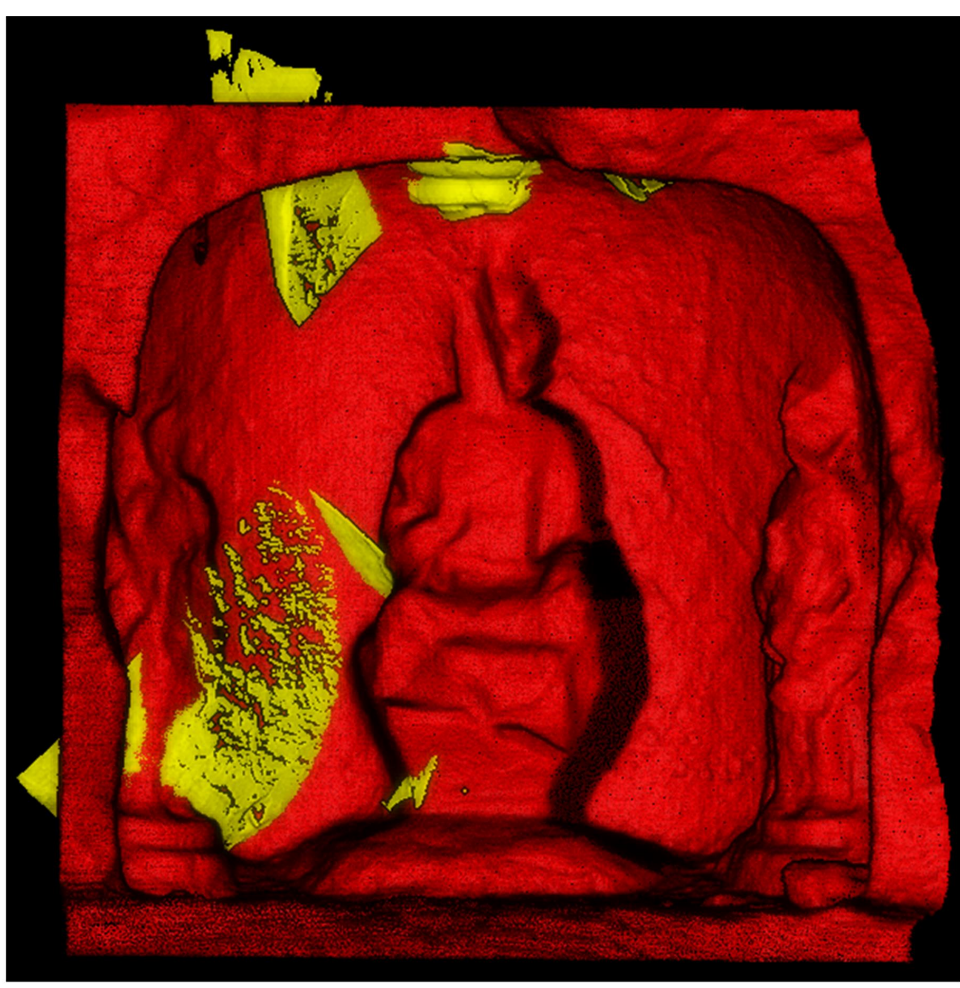

(b)

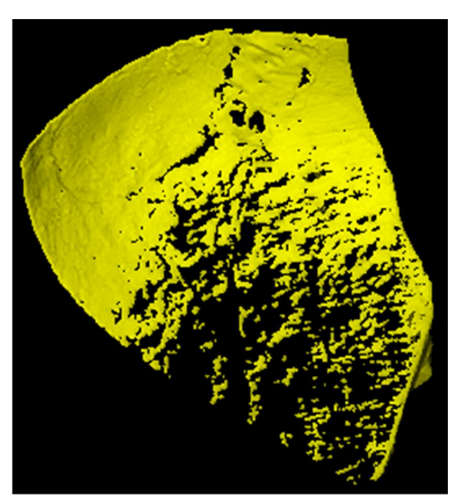

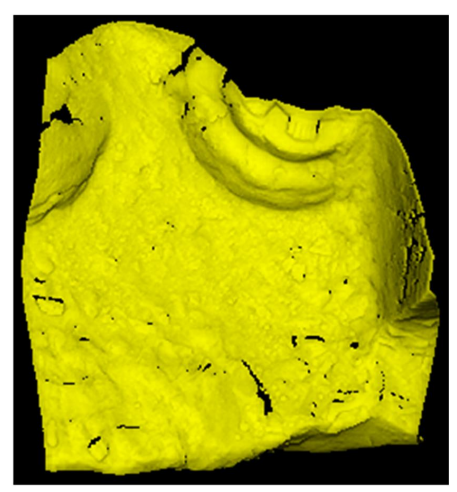

(c) 


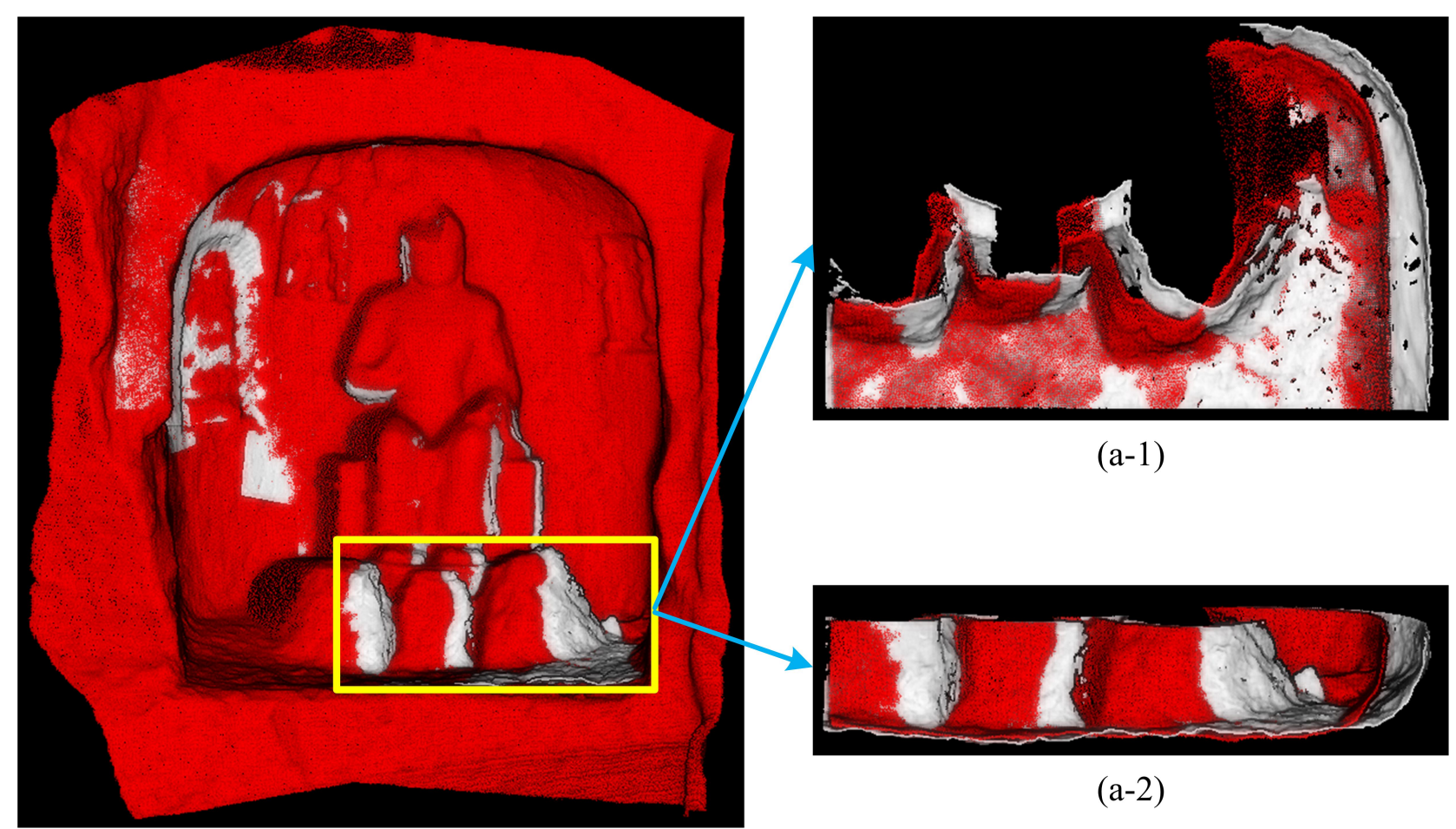

(a)

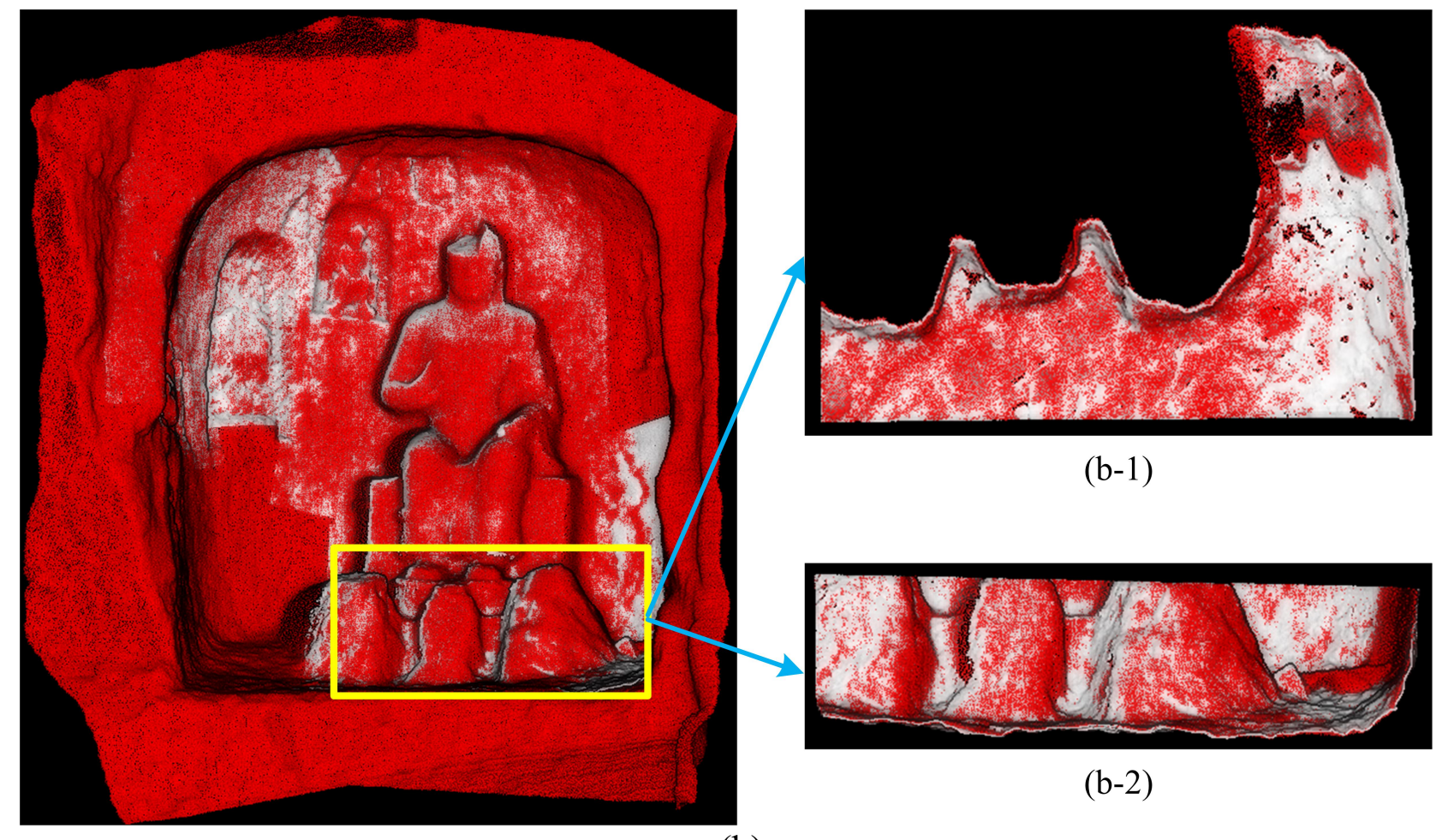

(b) 